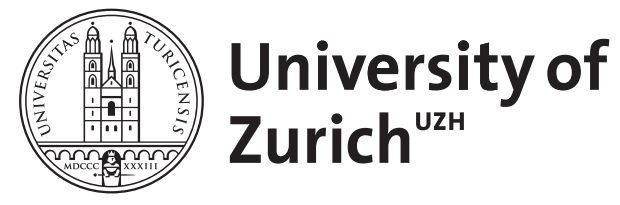
Archive

University of Zurich

University Library

Strickhofstrasse 39

CH-8057 Zurich

www.zora.uzh.ch

Year: 2012

Explaining brain size variation: from social to cultural brain

van Schaik, C P ; Isler, K ; Burkart, J M

DOI: https://doi.org/10.1016/j.tics.2012.04.004

Posted at the Zurich Open Repository and Archive, University of Zurich

ZORA URL: https://doi.org/10.5167/uzh-65158

Journal Article

Published Version

Originally published at:

van Schaik, C P; Isler, K; Burkart, J M (2012). Explaining brain size variation: from social to cultural brain. Trends in Cognitive Sciences, 16(5):277-284.

DOI: https://doi.org/10.1016/j.tics.2012.04.004 


\title{
Explaining brain size variation: from social to cultural brain
}

\author{
Carel P. van Schaik, Karin Isler and Judith M. Burkart
}

Anthropological Institute \& Museum, University of Zurich, Winterthurerstrasse 190, CH-8057 Zurich, Switzerland

\begin{abstract}
Although the social brain hypothesis has found nearuniversal acceptance as the best explanation for the evolution of extensive variation in brain size among mammals, it faces two problems. First, it cannot account for grade shifts, where species or complete lineages have a very different brain size than expected based on their social organization. Second, it cannot account for the observation that species with high socio-cognitive abilities also excel in general cognition. These problems may be related. For birds and mammals, we propose to integrate the social brain hypothesis into a broader framework we call cultural intelligence, which stresses the importance of the high costs of brain tissue, general behavioral flexibility and the role of social learning in acquiring cognitive skills.
\end{abstract}

\section{The social brain}

It has long been known that various mammalian and bird lineages differ in brain size, relative to body size [1,2]. The most widely accepted explanation is known as the Machiavellian intelligence [3] or social brain hypothesis [4]. Developed to explain variation order in relative brain size among mammalian orders and within primates, the hypothesis argues that large brains are adaptations for dealing with the complexities of social life. Several studies have documented the remarkably sophisticated understanding of the social world in primates [5], as well as some birds [6]. Although the originally used adjective 'Machiavellian' seemed to stress the competitive aspects of social life, the hypothesis equally applies to cooperative aspects, such as social learning, cooperation and coordination [7,8].

The social brain hypothesis is also consistent with the unusually large brain size of humans. In contrast to all other nonhuman primates, humans have evolved novel socio-cognitive abilities that build on a fully-fledged theory of mind and specialized social learning skills [9]. They arise early in ontogeny [10] and precede the development of physical and spatial cognitive abilities [11]. The most important among these abilities is language, which critically relies on shared intentionality, cooperative communicative intent and the attribution of mental states to others [12].

Broad comparative analyses support a prominent role of social interactions in the evolution of cognition in primates, other mammals and birds. The relative size of a species' brain or neocortex is correlated with the size of its social network [13] or the presence of coalitions and strong social bonds, including intrasexual pair bonds [8,14]. Indeed, being able to establish and maintain close social bonds is adaptive [15-17]. Consequently, mammalian lineages with stable social groups have shown far steeper increases in brain size over time than solitary ones $[14,18]$.

Despite this compelling support for the social brain hypothesis, two significant problems remain. First, it does not explain the existence of grade shifts, in that some lineages exhibit equally high socio-cognitive abilities with much smaller brains, both within primates [19] and other mammals [20], and most spectacularly, fish [21]. Second, recent studies suggest that primates and some other organisms show evidence for general intelligence, and it is not immediately obvious how selection on modular cognitive abilities (i.e., abilities limited to a specific domain) could have produced more general cognitive abilities. We will discuss these problems below and suggest that they are related. We then consider a broader hypothesis for birds and primates that encompasses the social brain hypothesis.

\section{Grade shifts and the social brain}

For some species or lineages, the social brain hypothesis does a poor job of predicting their brain size. On the one hand, orangutans (Pongo spp.) or aye-ayes (Daubentonia madagascariensis) live in socially simple societies but nonetheless have larger brains than related primates living in far more complex societies [19]. On the other hand, some relatively small-brained taxonomic groups have better sociocognitive abilities than expected on the basis of their brain size, a phenomenon known as grade shifts. For instance, lemurs achieve similar social complexity at smaller brain size than monkeys $[13,22]$. Comparisons of primates with other lineages provide more striking examples. Spotted hyenas (Crocuta crocuta) are relatively small-brained but show socio-cognitive abilities and a social organization very similar to that of the baboons with whom they coexist [20], and even tiny-brained Bechstein bats (Myotis bechsteinii) manage to live in primate-like fission-fusion societies with complex relationship dynamics [23]. When the comparison is extended to non-mammal and non-bird species, the discrepancies become even more marked. Several small fish species show socio-cognitive abilities rivaling those of primates $[21,24]$, despite having relatively minute brains. (Because the fish case can be linked to the modularity debate, its discussion is postponed until the next section.)

Traditionally, cognitive abilities are estimated using relative brain sizes, which are obtained by removing the effects of body size on brain size by taking residuals from 
an empirical regression curve or calculating encephalization quotients (a measure of relative brain size defined as the ratio between actual brain mass and predicted brain mass) [1]. Recent work, however, suggests that only a modest correction for body size is warranted [25,26]. Nevertheless, regardless of whether we use absolute or relative brain size, these grade shifts remain striking. Why, therefore, are some taxa systematically larger- or smaller-brained than expected based on the social brain hypothesis?

This could of course merely indicate that non-social challenges exist as well. Indeed, recent primate data suggest that ecological challenges are also important. These range from the ever-changing distribution of resources, which requires orientation and spatio-temporal memory abilities and some degree of planning of range use [27,28], to technical aspects of foraging, including tool use $[19,29,30]$, to the presence of complex anti-predator strategies [31]. However, among primates these non-social cognitive abilities are mostly found in the same species that excel in the socio-cognitive ones [26,32], and thus do not explain much additional variation.

Grade shifts in the relative brain size of birds and mammals can be explained in at least two ways. First, there may be physical constraints on the size of brains. These may reflect the need for morphological integration of the cranium and the masticatory apparatus, but probably the most common one is imposed by small body size. Because brain size shows negative allometry (that is, a less than isometric relationship of brain size to shape, anatomy and physiology) [1], in small-bodied species the brain constitutes a larger proportion of body mass, which implies that space is limited for both sense organs and brains [33]. Other constraints may be more particular. Bats have fairly small brains and it has been argued that they are under strong energetic constraints due to high costs of flight [34].

The second explanation for the presence of grade shifts in brain size refers to the fact that brains are energetically so expensive that organisms may have difficulty garnering the energy needed to maintain themselves $[33,35,36]$. This argument is part of a more general explanation that invokes the presence of a life-history filter for cognitive adaptation [35]. This filter (see legend of Figure 1) is a result of the high energetic and developmental costs of brains. To clarify why this should be the case, we must first consider selection on larger brain size, which in turn explains why the costs of larger brains must be met through changes in life history.

Natural selection favors an increase in the size of some organ if this increases lifetime reproductive success by

\section{Box 1. How organisms pay for increased brain size}

The high energy costs of brains mean that increased encephalization from a given ancestral state is possible only when extra energy is made available. This can be achieved along two fundamental, complementary pathways [64]: a stable increase in energy inputs (i.e. an increase in the mean, a reduction in the variance, or both) and redirection of energy away from other functions without any change in the overall net energy intake (Figure I). With respect to the first pathway, increased energy inputs correlated with brain enlargement have been found to come from allomaternal care [65], a richer diet or more effective foraging strategies (e.g. [66]), or from cognitive buffering [67] of environmental seasonality [68]. As expected, the species involved show a positive correlation between basal metabolic rate and brain size in placental [69] and marsupial [65] mammals. Concerning the second pathway, among the various possibilities (cf. [70]), only the tradeoff between brain size and 'production' (the combination of growth and reproduction) is empirically supported $[64,70,71]$. Reflecting this tradeoff, larger-brained mammals mostly have slower development $[70,72]$.

Regardless of the pathway, for selection to favor a brain size increase, the latter must improve adult survival, reproduction, or both. In practice, we usually see lower rates of reproduction $[70,73]$, perhaps largely because larger-brained species have heavier neonates [70]. The alternative, a compensatory increase in adult lifespan, is confirmed by many studies [70,74,75]. Consequently, because unavoidable mortality ultimately determines adult lifespan [39], the external conditions (predation, disease, seasonality) must allow for such an increase in adult lifespan. Otherwise, selection cannot favor a brain size increase.

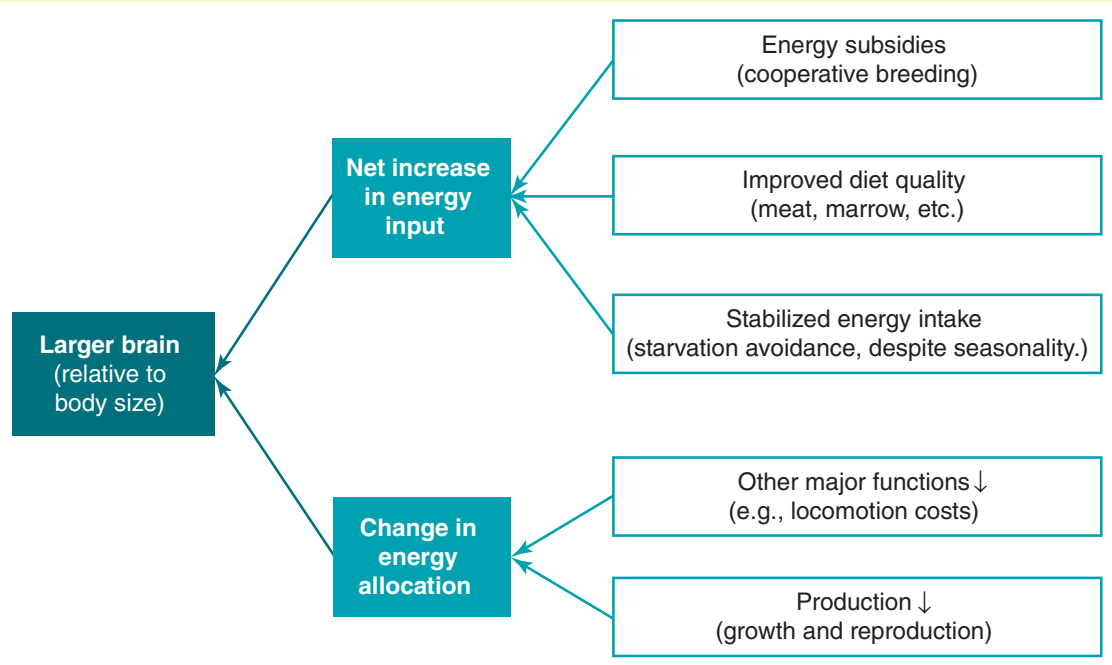

$\overline{\text { TRENDS in Cognitive Sciences }}$

Figure I. The major pathways followed by evolution to pay for the increased costs when brains get bigger (after [64,70]). 
improving the rate of survival or reproduction. Strictly speaking, of course, this is a net improvement, after the costs of the increase have been met. Normally, we can ignore these costs, but in the case of brain tissue they are not trivial, because brain tissue is energetically costly to maintain in terms of calories per unit weight and time, especially when it is still growing and differentiating. Moreover, its energy needs cannot be temporarily reduced [37,38], preventing a flexible resource allocation over time as is possible for other functions or tissues, such as somatic growth or muscle.

Empirical data show that these energetic and lifehistory costs of increased brain size are mainly met through an increase in adult lifespan (Box 1). This finding provides a second explanation for the presence of grade shifts in relative brain size. Natural selection will gradually turn improved adult survival into slower-paced life history [39], but only if high unavoidable mortality imposed by another factor does not prevent it. It is therefore possible that those lineages that do not have larger brains were unable to translate the potential cognitive benefits into sufficiently improved survival because external factors kept unavoidable mortality at a high level by acting as a lifehistory filter (Figure 1). Where this happens, brains remain stuck at a particular size, even if potential cognitive benefits would accrue from increased brain size. This problem is probably unique to brains because in other traits, the costs of development and maintenance are not so acute.

Here, we highlight two possible causes of the operation of a life-history filter. First, because brains have constant and high energy needs, seasonality may constrain the evolution of larger brains. Cognitive buffering of fluctuations in food availability, such as the flexible switching between different resources, may be impossible where seasonality is too severe. Thus, lemurs may be condemned to be small-brained due to the presence of an extreme lean season in Madagascar, with the telling exception of the large-brained aye-aye, which extracts larvae from wood and experiences virtually no seasonality [40]. Second, predation may be unavoidable for small species that have many large predators. (Note that the body-size effect mentioned above may be compounded by this life-history effect.) Because primates, including apes, have a long history of arboreality and were thus subject to

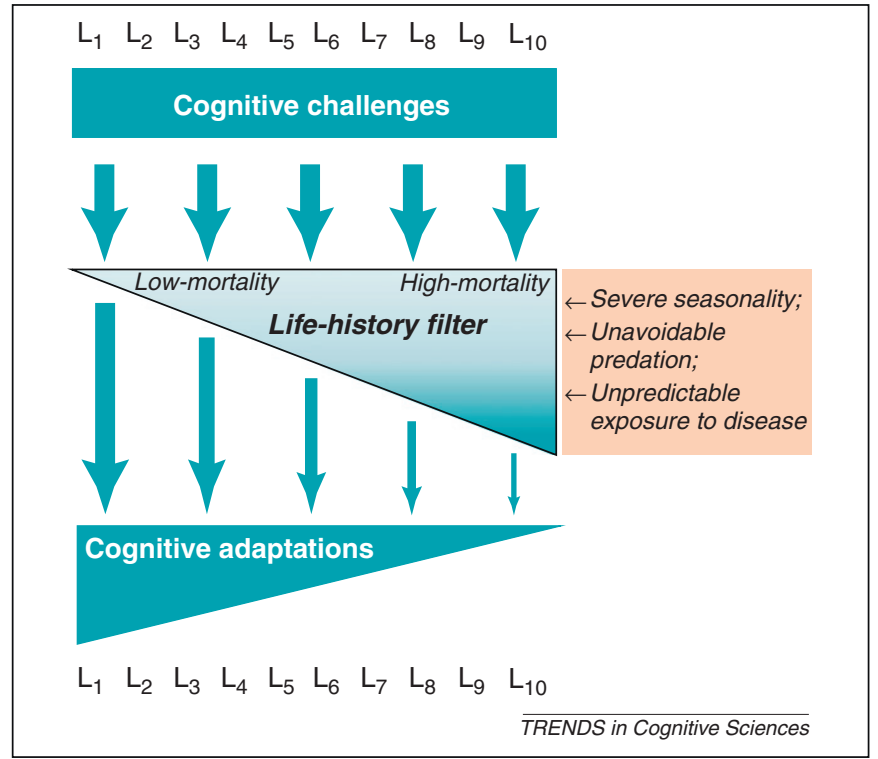

Figure 1. The life-history filter illustrated for 10 different lineages $\left(L_{1}\right.$ through $\left.L_{10}\right)$ sorted by rate of unavoidable mortality. Cognitive challenges, regardless of domain, may give rise to cognitive adaptations only to the extent that unavoidable mortality allows the slowdown in life-history pace required to support the requisite increase in brain size.

lower predation rates than terrestrial mammals of similar body size [35,41], they could afford to evolve larger brains. Thus, the life-history filter produces grade shifts because environmental conditions that determine unavoidable mortality prevent potential improvements due to cognitive solutions from being expressed.

The idea that small body size or fast life history impose limitations on brain size implicitly assumes that most lineages would benefit from increasing cognitive abilities, be they domain-specific or -general. This is not implausible because most species do not simply respond to ecological or social challenges but in part create them as well through niche construction [42]. The life-history filter also provides the best available answer yet to the age-old question why the majority of species are not 'clever' when they would derive such obvious benefits from it.

The presence of a filter due to body size or life history, and hence grade shifts, per se is not incompatible with the social brain hypothesis. However, what is incompatible is

\section{Box 2. General intelligence in nonhumans}

The observation that in humans performance across very different cognitive domains is correlated (the positive manifold) and that one single factor can explain a significant amount of this variation, is recognized in the term 'general intelligence' or ' $g$ '. The latter is highly heritable in humans, with genetic differences accounting for $40 \%-$ $50 \%$ of variance $[76,77]$, correlated with brain size [78] and a variety of life outcomes, such as school achievement, occupational attainment, job performance and even health and survival (see [79], for a review). Psychologically, g can be linked to processes such as the size of working memory, attention span, processing speed and inhibitory control over responses (for a review, see [76]). The presence of $g$ does not imply that individuals may not have cognitive domains in which they show particular strengths or weaknesses, but such individual differences in broad cognitive domains contribute a small amount of variance compared with $\mathrm{g}$ [79]. Indeed, the role of $\mathrm{g}$ is so pervasive that attempts to identify specific cognitive abilities neurobiologically often have to control statistically for its confounding effect [76].

Although domain-general cognitive abilities are often considered unique to humans, they are not. Across primate species, comparative studies have found that the performance of individual species on different cognitive tasks, involving aspects of spatial, physical and social cognition, is highly correlated $[25,26,32,80]$, suggesting the presence of a general factor underlying this variation. They have also found that this general cognitive performance is correlated quite strongly with brain size, corrected slightly for the effects of body size $[25,26]$. Similar intraspecific studies on cotton-top tamarins (Saguinus oedipus) support the notion of general intelligence [81], whereas results on chimpanzees also agree if we accept the finding [82] that spatial cognition is on a separate dimension. Domain-general cognitive abilities may be even more widespread in the animal kingdom and also occur in other mammals and birds [83-85]. They are particularly well documented in rodents, which also provide some insight into the evolutionary continuity of the mechanism. For instance, like in humans, g-factors extracted in rodent studies covary with efficacy in selective attention and working memory capacity $[86,87]$. 
the fact that species living in simple social systems, such as orangutans, aye-ayes or bears [20] have large brains. Thus, we need a hypothesis that can also explain the second kind of grade shifts.

\section{Domain-specific or domain-general cognitive abilities?}

The second problem with the social brain hypothesis is the growing evidence for a non-modular, general intelligence: an overarching ability to respond flexibly to new or complex situations, to learn and to innovate. Studies reviewed in Box 2 support the idea that general intelligence is not a uniquely derived human trait but instead a phylogenetically old phenomenon, found among primates, rodents and birds.

Parallel emergence of abilities in multiple domains is not explained by the social brain hypothesis or any other hypothesis focusing on the adaptive benefits of cognitive performance in a single domain unless selection on any domain-specific ability automatically produces general cognitive abilities [13,43]. However, we know that this is not universally true, as shown by the existence of modular abilities, such as food caching in birds, tactical deception in small fishes, tool use in ants or social communication in bees $[21,44]$. Moreover, if it were true, we would never know in which domain selection has favored the cognitive abilities. Worse, we would not even know whether the initial selection was on domain-specific cognitive abilities or on domain-general ones.

More plausible, therefore, is the idea that selection actually favored domain-general cognitive abilities that could be applied to whichever problems a developing individual would face. Applying such abilities predominantly in one domain, say social cognition, may lead to automatization of skills. This process could be called secondary modularization because it produces many of the features commonly associated with modules rather than domaingeneral reasoning, such as fast and frugal processing and independence of reasoning. In humans, this idea is relatively uncontroversial, since no one would suggest that we have an evolved module for driving a car or playing chess [45]. However, some evidence suggests automatization may also occur in nonhumans. Thus, in primates, a marked difference in tool use ability between wild and captive populations is well documented, in some species to the point that tool use has been exclusively documented in captivity $[29,46]$. Yet, once proficient, such individuals show tool use with high degrees of automatization. Secondary modularization is also supported by plastic changes in the brain, such as the changes in gray matter content of parts of the neocortex of rhesus monkeys in response to changes in social group size [47].

Domain-general cognitive abilities and domain-specific socio-cognitive abilities can, of course, coexist in the same species. This raises the question how their relative importance varies taxonomically. It seems reasonable to assume that small-brained organisms cannot afford to support domain-general cognitive abilities. Indeed, evidence available so far is consistent with the idea that invertebrates and poikilothermic vertebrates, with relatively smaller brains $[1,33,36,48]$, do not show much of them [21]. On the other hand, domain-general abilities are found in both birds and mammals, whose brains are an order of magnitude larger for their body size than those of fishes, amphibians or reptiles $[33,36]$. Thus, one could speculate that the major expansion of integrative brain structures, and thus large brain size [49], which arose independently in these lineages, was at least partly linked to the addition of a domain-general learning and action-planning ability.

We expect that most birds and mammals have a mix of secondary modularization and classic modularity, with innate preferences for particular kinds of input, independent from other learning or attention processes [44]. Variation among great ape species in innovation and problem-solving biases illustrates this. Thus, bonobos bias their innovations, including those involving tool use, more toward solving social problems, chimpanzees more toward solving subsistence problems, and orangutans more toward enhancing physical comfort [50-53]. These divergent biases are found in the wild, but also in captivity, where conditions are uniform across the species concerned.

We can now also offer another speculative but testable explanation of some of the apparent grade shifts in the relation between brain size and socio-cognitive abilities. The more organisms rely on domain-specific socio-cognitive adaptations, the more they may be able to perform these tasks with relatively small brains. In extreme cases, domain-general abilities trained on socio-cognitive challenges may be absent, such as perhaps in cleaner fishes [21], whereas at the other extreme, perhaps, for instance, in humans, the dedicated modules play a minor role. This prediction can be tested by examining the species concerned for evidence of domain-general cognitive abilities (Box 2).

\section{A cultural rather than just social brain}

The existence of domain-general cognitive abilities in larger-brained birds and primates is incompatible with the social brain hypothesis. However, it is entirely consistent with Reader and Laland's [32] proposal that general behavioral flexibility, not tied to any domain in particular (i.e. content-neutral), is the adaptation underlying cognitive performance in primates. For some reason, however, this idea did not become as popular as the social brain hypothesis, despite its strong explanatory power.

The behavioral flexibility hypothesis itself, however, also faces an objection. A general problem-solving ability may be adaptive by allowing initially naive individuals to develop a set of customized skills to handle specific local environmental challenges. However, it would be difficult for selection to promote it beyond a fairly modest level. The reason is that, in the absence of transmission to the next generation through social learning, the invented skills will merely provide a one-off fitness advantage to the inventor. The rare innovations that reflect a species' behavioral flexibility [32] and allow it to cope with environmental change [54] greatly improve fitness but are very unlikely to arise again independently in the inventor's offspring. Worse, even routine foraging, parenting or mating skills shown by any normal adult of a given species are often not spontaneously invented by maturing individuals, and only acquired when they are exposed to role models (for a review, see [55]). Innovations must therefore be made heritable by social learning [55]. 
Social learning, favored by kin selection, goes beyond making innovations heritable. It is also a much faster and much more reliable way of acquiring solutions than asocial exploration and learning, making it an energetically vastly more efficient use of expensive brain tissue. Finally, selection on more effective techniques of social learning also automatically improves the asocial learning ability because of the high cognitive overlap between the two [56]. This overlap also ensures correlated evolution between innovative and social-learning abilities. These ideas derive from the cultural intelligence hypothesis $[7,55,57]$. The hypothesis proposes that general cognitive ability, and thus brain size, can increase where association with tolerant role models is sufficiently high that skills of any kind can be learned faster and innovative skills be learned at all. It combines elements of the behavioral flexibility [26,32] and Vygotskian intelligence hypotheses $[58,60]$ that emerged alongside the social brain hypothesis (see also [59]). It proposes a content-neutral (domain-general) adaptation to learn skills socially and thus also asocially, at least for larger birds and mammals. The learned skills acquired can be social, ecological or spatial, even though in

\section{Box 3. Cultural intelligence in nonhumans?}

Cultural intelligence refers to the developmental construction of general learning abilities and cognitive skills (potentially biased toward particular domains). This construction takes place under the influence of social role models, who structure attention and serve as imitation targets, possibly in interaction with innate preferences for particular kinds of inputs of varying content and strength in different species. It is therefore a Vygotskian perspective [58] applied to nonhuman animals.

The cultural intelligence hypothesis largely predicts the same interspecific patterns in brain size as the social brain hypothesis, but it alone predicts that cognitive abilities, proxied by brain size, should reflect the duration of availability or the number of tolerant role models for social learning. As shown in Figure I, a first round of empirical testing [55] supported this prediction for birds and solitary carnivorous mammals.

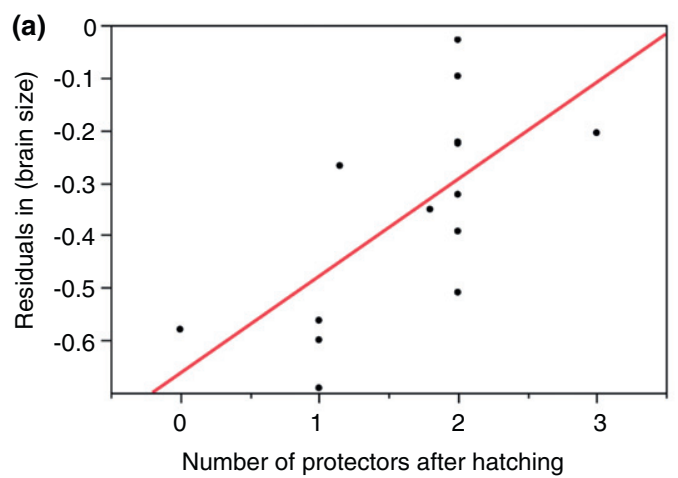

Also consistent with the cultural intelligence hypothesis is work showing variation in space and time in primate cognitive skills among nonhuman species, reflecting the role of social learning in acquiring cognitive skills:

- Cultural variation among wild populations of great apes in cognitively complex skills $[7,88]$.

- Major differences between captive and wild primates in the nature of cognitive competence [89-92].

- An effect of rearing conditions on cognitive performance in different domains (see $[55,93]$, for reviews).

- An effect of previous cultural experience on the ability to solve specific problems $[94,95]$.

- The finding that even routine skills, such as nest building or mothering behavior, are acquired through social learning (see [55], for a review).

- A major effect of cultural and educational differences on brain structure in apes and rodents [96], just as in humans [97].
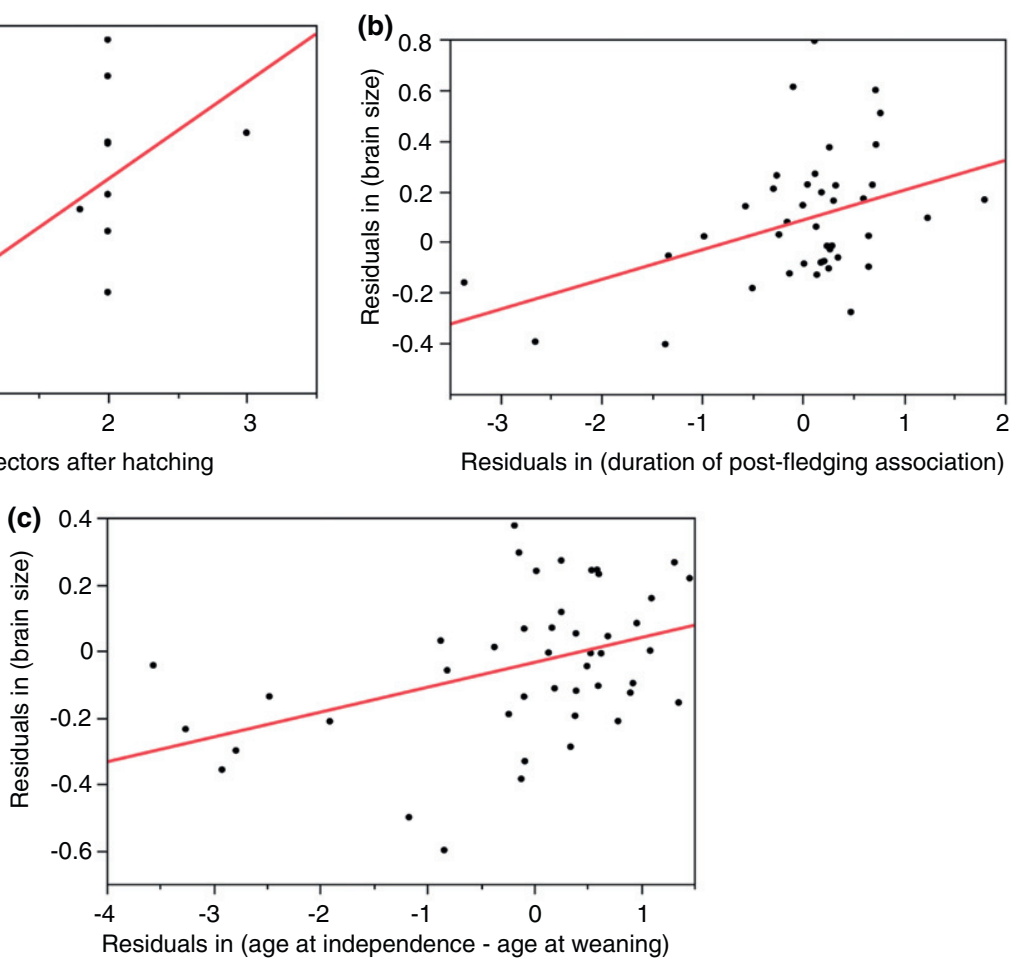

TRENDS in Cognitive Sciences

Figure I. (a) Mean values of residual (observed minus expected on the basis of body mass) brain size for species in precocial bird families ( $N=14$ ) as a function of the mean number of protectors after hatching, and thus opportunities for social learning. The correlation is significant $\left(p=0.009 ; \mathrm{r}^{2}=0.447\right)$. Family means are used, because in birds variation in brain size is found mainly at this level [98]. Data from Karin Isler's database compiled from numerous sources (especially [99-103]). (b) Mean values of residual (observed minus expected on the basis of body mass) brain size for species in altricial bird families ( $\mathrm{N}=42$ ) as a function of the residual duration of post-fledging association with parents, and thus opportunities for social learning. The correlation is significant $\left(p=0.007 ; r^{2}=0.171\right)$. (c) Residual brain size of solitary carnivore species $(\mathrm{N}=45)$ as a function of the residual of the duration of post-weaning association with the mother, which reflects the frequency of opportunities for social skill learning. The correlation is significant for species values $(p=0.004)$ and remains so for independent contrasts $(p=0.02)$. Data from compilations $[104,105]$. 
many species the skills acquired socially may have a content bias toward the social domain.

Grade shifts can still arise, of course, and for the same reasons as discussed above. However, an additional cause now also comes into play: variation in the frequency of opportunities for social transmission across generations. Indeed, this is one way to distinguish the cultural intelligence hypothesis from the social brain hypothesis, with which it shares virtually all other predictions because social life also allows skill learning that is socially mediated through association, observation or interaction. Uniquely, the cultural intelligence hypothesis predicts that animals that do not live in stable social groups as adults, but in which immatures associate with adults, should also be able to have evolved larger brains. Box 3 presents an empirical test confirming this prediction [55] and reviews other phenomena consistent with this hypothesis.

This idea is also consistent with what we know about human intelligence, and thus parsimoniously assumes evolutionary continuity between humans and nonhumans (Box 2). Indeed, cultural intelligence can also account for the unusual brain size increase in human evolution, where high social tolerance turned into active skill transmission, engendered by the adoption of cooperative breeding and the evolution of teaching $[61,62]$. Thus, a positive feedback cycle arose, in which ever-growing reliance on technological subsistence skills transmitted across generations selected for improved social-learning abilities and thus indirectly for improved innovative abilities [63].

\section{Concluding remarks}

In conclusion, we noted two weaknesses in the social brain hypothesis: it cannot account for the presence of domaingeneral cognitive abilities in birds and mammals, and it leaves grade shifts in brain size unexplained.

Unexplained grade shifts can have thee causes. First, some filter (e.g., due to small body size or high unavoidable mortality) may prevent the evolution of a larger brain, even though selection would in principle favor improved sociocognitive abilities. Second, the taxon's high socio-cognitive abilities are entirely or largely based on domain-specific socio-cognitive adaptations, which may hardly affect overall brain size. We speculated that animals without domaingeneral cognitive abilities are mainly small-brained, perhaps because they are affected by such filters. Third, some have large brains despite living in simple societies.

The cultural intelligence hypothesis recognizes that in some lineages domain-general cognitive abilities evolved that could be trained especially on socio-cognitive challenges, in addition to the specialized cognitive adaptations already present. In these animals, maturing individuals acquire vital cognitive skills, be they social or ecological, through social learning. This idea makes many of the same predictions as the social brain hypothesis but can also account for the third kind of grade shifts left unexplained by the latter. Obviously, it applies only to those species where domain-general cognitive abilities are well developed.

\section{Acknowledgments}

The research reported here was supported by the Swiss National Fund (SNF-grant nos 105312-114107, 31003A-111915, 31003A-117789 and
310030-13083). We thank Sonja Koski, Caroline Schuppli, Michèle Schubiger and three anonymous reviewers for valuable comments.

\section{References}

1 Jerison, H.J. (1973) Evolution of Brain and Intelligence, Academic Press

2 Lefebvre, L. et al. (2004) Brains, innovations and evolution in birds and primates. Brain Behav. Evol. 63, 233-246

3 Byrne, R.W. and Whiten, A. (1988) Machiavellian Intelligence: Social Expertise and the Evolution of Intellect in Monkeys, Apes and Humans, Calderon Press

4 Dunbar, R.I.M. (1998) The social brain hypothesis. Evol. Anthropol. 6, $178-190$

5 Silk, J. (2007) The adaptive value of sociality in mammalian groups. Philos. Trans. R. Soc. B: Biol. Sci. 362, 539-559

6 Emery, N. et al. (2007) Cognitive adaptations of social bonding in birds. Philos. Trans. R. Soc. B: Biol. Sci. 362, 489-505

7 Whiten, A. and van Schaik, C.P. (2007) The evolution of animal 'cultures' and social intelligence. Philos. Trans. R. Soc. B: Biol. Sci. $362,603-620$

8 Dunbar, R.I.M. and Shultz, S. (2007) Evolution in the social brain. Science 317, 1344-1347

9 Gergely, G. et al. (2007) On pedagogy. Dev. Sci. 10, 139-146

10 Baillargeon, R. et al. (2010) False-belief understanding in infants. Trends Cogn. Sci. 14, 110-118

11 Herrmann, E. et al. (2007) Humans have evolved specialized skills of social cognition: the Cultural Intelligence Hypothesis. Science 317, 1360-1366

12 Tomasello, M. (2008) Origins of Human Cooperation, MIT Press

13 Dunbar, R.I.M. (2003) The social brain: mind, language, and society in evolutionary perspective. Annu. Rev. Antrhopol. 32, 163-181

14 Shultz, S. and Dunbar, R.I.M. (2010) Social bonds in birds are associated with brain size and contingent on the correlated evolution of life history and increased parental investment. Biol. J. Linn. Soc. 100, 111-123

15 Silk, J.B. et al. (2009) The benefits of social capital: close social bonds among female baboons enhance offspring survival.Proc. R. Soc. B 276, 3099-3104

16 Silk, J.B. et al. (2010) Strong and consistent social bonds enhance the longevity of female baboons. Curr. Biol. 20, 1359-1361

17 Seyfarth, R.M. and Cheney, D.L. (2012) The evolutionary origins of friendship. Annu. Rev. Psychol. 63, 153-177

18 Shultz, S. and Dunbar, R.I.M. (2010) Encephalization is not a universal macroevolutionary phenomenon in mammals but is associated with sociality. Proc. Natl. Acad. Sci. U.S.A. 107, 21582 21586

19 Byrne, R.W. (1997) Machiavellian intelligence. Evol. Anthropol. 5, $172-180$

20 Holekamp, K. (2007) Questioning the social intelligence hypothesis Trends Cogn. Sci. 11, 65-69

21 Bshary, R. et al. (2002) Fish cognition: a primate's eye view. Anim. Cogn. 5, 1-13

22 Fichtel, C. and Kappeler, P.M. (2009) Human universals and primate symplesiomorphies: establishing the lemur baseline. In Mind the Gap: Tracing the Origins of Human Universals (Kappeler, P.M. and Silk, J., eds), pp. 395-426, Springer

23 Kerth, G. et al. (2011) Bats are able to maintain long-term social relationships despite the high fission-fusion dynamics of their groups. Proc. R. Soc. B 278, 2761-2767

24 Pinto, A. et al. (2011) Cleaner wrasses Labroides dimidiatus are more cooperative in the presence of an audience. Curr. Biol. 21 , 1140-1144

25 Deaner, R.O. et al. (2007) Overall brain size, and not encephalization quotient, best predicts cognitive ability across non-human primates. Brain Behav. Evol. 70, 115-124

26 Reader, S.M. et al. (2011) The evolution of primate general intelligence. Philos. Trans. R. Soc. B: Biol. Sci. 366, 1017-1027

27 Normand, E. et al. (2009) Forest chimpanzees (Pan troglodytes verus) remember the location of numerous fruit trees. Anim. Cogn. 12, 797807

28 Zuberbühler, K. and Janmaat, K. (2010) Foraging cognition in nonhuman primates. In Primate Neuroethology (Platt, M.L. and Ghazanfar, A.A., eds), pp. 64-83, Oxford University Press 
29 van Schaik, C.P. et al. (1999) The conditions for tool use in primates: implications for the evolution of material culture. J. Hum. Evol. 36, 719-741

30 Sanz, C.M. and Morgan, D.B. (2007) Chimpanzee tool technology in the Goualougo Triangle, Republic of Congo. J. Hum. Evol. 52, $420-433$

31 Zuberbühler, K. and Byrne, R.W. (2006) Social cognition. Curr. Biol. 16, R786-R790

32 Reader, S.M. and Laland, K.N. (2002) Social intelligence, innovation and enhanced brain size in primates. Proc. Natl. Acad. Sci. U.S.A. 99, 4436-4441

33 Striedter, G.F. (2005) Principles of Brain Evolution, Sinauer Associates

34 Safi, K. et al. (2005) Bigger is not always better: when brains get smaller. Biol. Lett. 1, 283-286

35 van Schaik, C.P. and Deaner, R.O. (2003) Life history and cognitive evolution in primates. In Animal Social Complexity (de Waal, F.B.M. and Tyack, P.L., eds), pp. 5-25, Cambridge, MA, Harvard University Press

36 Allman, J.M. (1999) Evolving Brains, Scientific American Library

37 Karasov, W. et al. (2004) Anatomical and histological changes in the alimentary tract of migrating blackcaps (Sylvia atricapilla): a comparison among fed, fasted, food-restricted, and refed birds. Physiol. Biochem. Zool. 77, 149-160

38 Bauchinger, U. et al. (2005) Flexible remodeling of organ size during spring migration of the garden warbler (Sylvia borin). Zoology (Jena) 108, 97-106

39 Stearns, S.C. et al. (2000) Experimental evolution of aging, growth, and reproduction in fruitflies. Proc. Natl. Acad. Sci. U.S.A. 97, 33093313

40 van Woerden, J.T. et al. (2010) Effects of seasonality on brain size evolution: evidence from strepsirrhine primates. Am. Naturalist 176 , 758-767

41 Shattuck, M.R. and Williams, S.A. (2010) Arboreality has allowed for the evolution of increased longevity in mammals. Proc. Natl. Acad. Sci. U.S.A. 107, 4635-4639

42 Laland, K.N. et al. (2000) Niche construction, biological evolution and cultural change. Behav. Brain Sci. 23, 131-175

43 Byrne, R.W. and Bates, L.A. (2007) Sociality, evolution and cognition. Curr. Biol. 17, R714-R723

44 Shettleworth, S.J. (2010) Cognition, Evolution and Behavior, (2nd ed.), Oxford University Press

45 Chi, M.T.H. (2006) Laboratory methods for assessing experts' and novices' knowledge. In The Cambridge Handbook of Expertise and Expert Performance (Ericsson, K.A. et al., eds), pp. 167-184, Cambrdige University Press

46 Shumaker, R.W. et al. (2011) Animal Tool Behavior: The Use and Manufacture of Tools by Animals, Johns Hopkins University Press

47 Sallet, J. et al. (2011) Social network size affects neural circuits in macaques. Science 334, 697-700

48 Wehner, R. et al. (2007) On being small: brain allometry in ants. Brain Behav. Evol. 69, 220-228

49 Finlay, B.L. et al. (2001) Developmental structure in brain evolution. Behav. Brain Sci. 24, 263-308

50 Herrmann, E. et al. (2010) Differences in the cognitive skills of bonobos and chimpanzees. PLoS ONE 5, e12438

51 van Schaik, C.P. et al. (2006) Innovation in wild Bornean orangutans (Pongo pygmaeus wurmbii). Behaviour 143, 839-876

52 Meulman, E.J.M. et al. (2012) The role of terrestriality in promoting primate technology. Evol. Anthropol. 21, 58-68

53 Gruber, T. et al. (2010) A comparison of bonobo and chimpanzee tool use: evidence for a female bias in the Pan lineage. Anim. Behav. 80, 1023-1033

54 Sol, D. et al. (2005) Big brains, enhanced cognition, and response of birds to novel environments. Proc. Natl. Acad. Sci. U.S.A. 102, 54605465

55 van Schaik, C.P. and Burkart, J.M. (2011) Social learning and evolution: the cultural intelligence hypothesis. Philos. Trans. $R$. Soc. Lond. B: Biol. Sci. 366, 1008-1016

56 Heyes, C. (2011) What's social about social learning? J. Comp. Psychol. http://dx.doi.org/10.1037/a0025180

57 van Schaik, C.P. (2006) Why are some animals so smart? Sci. Am. 294, $64-71$
58 Moll, H. and Tomasello, M. (2007) Cooperation and human cognition: the Vygotskian intelligence hypothesis. Philos. Trans. R. Soc. Lond. B: Biol. Sci. 362, 639-648

59 Wilson, A.C. (1991) From molecular evolution to body and brain evolution. In Perspectives on Cellular Regulation: From Bacteria to Cancer (Campisi, J. and Pardee, A.B., eds), pp. 331-340, J. Wiley \& Sons

60 Tomasello, M. (1999) The Cultural Origins of Human Cognition, Harvard University Press

61 Hrdy, S.B. (2009) Mothers \& Others: The Evolutionary Origins of Mutual Understanding, Harvard University Press

62 Burkart, J.M. et al. (2009) Cooperative breeding and human cognitive evolution. Evol. Anthropol. 18, 175-186

63 Sterelny, K. (2007) Social intelligence, human intelligence and niche construction. Philos. Trans. R. Soc. Lond. B: Biol. Sci. 362, 719-730

64 Navarrete, A. et al. (2011) Energetics and the evolution of human brain size. Nature 480, 91-93

65 Isler, K. (2011) Energetic trade-offs between brain size and offspring production: marsupials confirm a general amammalian pattern. Bioessays 33, 173-179

66 Leonard, W.R. and Robertson, M.L. (1997) Comparative primate energetics and hominid evolution. Am. J. Phys. Anthropol. 102, 265-281

67 Sol, D. (2009) Revisiting the cognitive buffer hypothesis for the evolution of large brains. Biol. Lett. 5, 130-133

68 van Woerden, J.T. et al. (2012) Large brains buffer energetic effects of seasonal habitats in caterrhine primates. Evolution 66, 191-199

69 Isler, K. and van Schaik, C.P. (2006) Costs of encephalization: the energy trade-off hypothesis tested on birds. J. Hum. Evol. 51, 228-243

70 Isler, K. and van Schaik, C.P. (2009) The expensive brain: a framework for explaining evolutionary changes in brain size. $J$. Hum. Evol. 57, 392-400

71 Barrickman, N.L. et al. (2008) Life history costs and benefits of encephalization: a comparative test using data from long-term studies of primates in the wild. J. Hum. Evol. 54, 568-590

72 Barton, R.A. and Capellini, I. (2011) Maternal investment, life histories, and the costs of brain growth in mammals. Proc. Natl. Acad. Sci. U.S.A. 108, 6169-6174

73 Isler, K. and van Schaik, C.P. (2009) Why are there so few smart mammals (but so many smart birds)? Biol. Lett. 5, 125-129

74 Sol, D. et al. (2007) Big-brained birds survive better in nature. Proc. $R$. Soc. B 274, 763-769

75 Gonzalez-Lagos, C. et al. (2010) Large-brained mammals live longer. J. Evol. Biol. 23, 1064-1074

76 Geary, D.C. (2005) The Origin of Mind: Evolution of Brain, Cognition, and General Intelligence, American Psychological Association

77 Davies, G. et al. (2011) Genome-wide association studies establish that human intelligence is highly heritable and polygenic. Mol. Psychiatry 16, 996-1005

78 McDaniel, M.A. (2005) Big-brained people are smarter: a metaanalysis of the relationship between in-vivo brain volume and intelligence. Intelligence 33, 337-346

79 Deary, I.J. et al. (2010) The neuroscience of human intelligence differences. Nat. Rev. Neurosci. 11, 201-211

80 Deaner, R.O. et al. (2006) Do some taxa have better domain-general cognition than others?. A meta-analysis of nonhuman primate studies. Evol. Psychol. 4, 149-196

81 Banerjee, K. et al. (2009) General intelligence in another primate: individual differences across cognitive task performance in a New World monkey (Saguinus oedipus). PLoS ONE 4, e5883

82 Herrmann, E. et al. (2010) The structure of individual differences in the cognitive abilities of children and chimpanzees. Psychol. Sci. 21, $102-110$

83 Emery, N.J. and Clayton, N.S. (2004) The mentality of crows: convergent evolution of intelligence in corvids and apes. Science 306, 1903-1907

84 Keagy, J. et al. (2011) Complex relationship between multiple measures of cognitive ability and male mating success in satin bowerbirds, Ptilonorhynchus violaceus. Anim. Behav. 81, 1063-1070

85 Lefebvre, L. (2011) Taxonomic counts of cognition in the wild. Biol. Lett. 7, 631-633

86 Matzel, L.D. et al. (2003) Individual differences in the expression of a 'general' learning ability in mice. J. Neurosci. 23, 6423-6433 
87 Matzel, L.D. et al. (2011) Individual differences in animal intelligence: learning, reasoning, selective attention and inter-species conservation of a cognitive trait. Int. J. Comp. Psychol. 24, 36-59

88 Byrne, R.W. (2007) Culture in great apes: using intricate complexity in feeding skills to trace the evolutionary origin of human technical prowess. Philos. Transact. R. Soc. B Biol. Sci. 362, 577-585

89 Halsey, L.G. et al. (2006) Can wild common marmosets (Callithrix jacchus) solve the parallel strings task? Anim. Cogn. 9, 229-233

90 Laidre, M.E. (2008) Spontaneous performance of wild babons on three novel food-access puzzles. Anim. Cogn. 11, 223-230

91 Russon, A.E. et al. (2010) How orangutans (Pongo pygmaeus) innovate for water. J. Comp. Psychol. 124, 14-28

92 Lehner, S.R. et al. (2010) An evaluation of the geographic method for recognizing innovations in nature, using zoo orangutans. Primates 51 , 101-118

93 Russell, J.L. et al. (2011) The role of socio-communicative rearing environments in the development of social and physical cognition in apes. Dev. Sci. 14, 1459-1470

94 Gruber, T. et al. (2009) Wild chimpanzees rely on cultural knowledge to solve an experimental honey acquisition task. Curr. Biol. 19, 1806-1810

95 van de Waal, E. and Bshary, R. (2010) Contact with human facilities appears to enhance technical skills in wild vervet monkeys (Chlorocebus aethiops). Folia Primatol. 81, 282-291
96 Sackett, G.P. et al. (1999) Early experience effects on adaptive behavior: theory revisited. Ment. Retard. Dev. Disab. Res. Rev. 5, $30-40$

97 Kitayama, S. and Park, J. (2010) Cultural neuroscience of the self: understanding the social grounding of the brain. Soc. Cogn. Affect. Neurosci. 5, 111-129

98 Nealen, P.M. and Ricklefs, R.E. (2001) Early diversification of the avian brain: body size relationship. J. Zool. 253, 391-404

99 Mlikovsky, J. (1989) Brain size in birds: 1. Tinamiformes through Ciconiformes. Acta Soc. Zool. Bohemoslov. 53, 33-47

100 Mlikovsky, J. (1989) Brain size in birds: 2. Falconiformes through Gaviiformes. Acta Soc. Zool. Bohemoslov. 53, 200-213

101 Mlikovsky, J. (1989) Brain size in birds: 3. Columbiformes through Piciformes. Acta Soc. Zool. Bohemoslov. 53, 252-264

102 Mlikovsky, J. (1990) Brain size in birds: 4. Passeriformes. Acta Soc. Zool. Bohemoslov. 54, 27-37

103 Iwaniuk, A.N. and Nelson, J.E. (2003) Developmental differences are correlated with relative brain size in birds: a comparative analysis. Can. J. Zool. 81, 1913-1928

104 Wilson, D.E. and Mittermeier, R.A. (2009) The Handbook of the Mammals of the World, Vol. 1: Carnivores, Lynx Ediciones

105 Gittleman, J.L. (1986) Carnivore life history patterns: allometric, phylogenetic, and ecological associations. Am. Nat. 127, 744-771 